\title{
ENHANCEMENT OF VALIDATION STUDIES FOR REACTOR DOSIMETRY AND ACTIVATION PREDICTIONS WITH THE NUCLEAR DATA SAMPLING METHODOLOGY
}

\author{
A. Vasiliev, M. Pecchia, D. Rochman, H. Ferroukhi \\ Paul Scherrer Institut \\ Forschungsstrasse 111, 5232 Villigen PSI, Switzerland \\ alexander.vasiliev@psi.ch, marco.pecchia@psi.ch, \\ dimitri-alexandre.rochman@psi.ch, hakim.ferroukhi@psi.ch
}

\begin{abstract}
The CASMO/SIMULATE/MCNP/FISPACT-II calculation route has been established at the Paul Scherrer Institute (PSI) for reactor dosimetry and activation studies. Furthermore, the in-house tool NUSS is in use at PSI for nuclear data (ND) related uncertainties quantifications with Monte Carlo neutron transport calculations. The use of randomly sampled ACE-formatted ND files not only allows propagation of the ND uncertainties, but also can serve for assessing the applicability of different types of experimental data for validation of calculation predictions of parameters of interest. In the present work an application of the PSI calculation scheme for analysis of activation reaction rates and the fast neutron fluence (FNF), throughout the Swiss pressurised water reactor (PWR) and simplified containment building models, is demonstrated. As particular examples of potentially available experimental data, two kinds of the neutron flux monitors are considered: a) the reactor pressure vessel scraping samples and detectors placed in the dosimetry channels, mounted at the core barrel and designed for validation of FNF calculations, and b) the exvessel dosimeters, specifically used by the Swiss waste management organisation (NAGRA) for validation of bio-shield activation predictions. The calculations were done with the ENDF/B-VII.1 library. The obtained results demonstrate importance of the ND uncertainties for the dosimetry evaluations. The assessment of the applicability of the selected experimental information for validation of the bio-shield irradiation calculations was done based on evaluation of the ND-related Pearson correlation coefficients.
\end{abstract}

KEYWORDS: Reactor dosimetry, Concrete bio-shield, Validation, Uncertainties, Correlations

\section{INTRODUCTION}

Two topics related to reactor neutronics and radiation transport simulations have received attention within the last decade worldwide. One is related to optimization of decommissioning and dismantling processes, which becomes important for the aging light water reactor (LWR) park of generation II nuclear power plants (NPPs). Another one is in turn related to the concepts of significantly extending the originally designed reactor lifetimes for some of the reactors from the same generation II fleet in some countries. Both topics are currently actual for the Swiss nuclear electricity sector. Therefore, two relatively new calculation tasks became important at PSI for supporting Swiss nuclear industry needs. The first is the $\mathrm{R} \& \mathrm{D}$ on reactor structures activation for efficient radioactive wastes characterization. The second is an 
enhanced prediction of the radiation doses received by reactor materials, for better assessment of the radiation-induced embrittlement, at the conditions of the extended reactor operation times. In the present paper, the recent developments realised at PSI towards simulation predictions in application to the two mentioned cases are outlined with primary focus given to the concrete reactor bio-shield (BS) irradiation.

The reactor concrete BS traditionally did not belong to the list of reactor structures for regular irradiation assessments and even the interest to the BS activation simulations has started to grow only recently, in relation to the forthcoming decommissioning projects [1,2]. It shall be recognised that on the contrary to the activation reactions, which are primary induced by thermal neutrons, the reactor materials (steels, concrete, reinforced concrete) degradation under irradiation is mainly caused by fast neutrons (see details, e.g., in [3]). Therefore, even though the both types of calculations have many parts in common, different sources of experimental data can be appropriate for rigorous validation of each specific calculation methodology. In this context, it shall be noted that the validation studies typically aim at assessment of calculation biases and uncertainties associated with usage of a particular computation code and, which may be most important, a particular ND library. Furthermore, it is practically relevant not only to propagate ND uncertainties in the calculation simulations, but also to assess correlations between an application system and available experimental benchmarks [4], which shall help selecting the most appropriate benchmarks for the methodology validation.

In order to address the above mentioned questions, the CASMO/SIMULATE/MCNP ${ }^{1} /$ FISPACT-II reference calculation methodology for LWR dosimetry applications, recently established at PSI [5,6], is now complemented with the capability for ND random sampling with the in-house tool NUSS [7]. The present work is done in application to a Swiss PWR reactor. The experimental data considered in this work includes: 1) the reactor pressure vessel 'scraping samples' and the 'gradient probes' detectors placed in the dosimetry channels, mounted at the core barrel and designed for validation of FNF calculations, and 2) the ex-vessel dosimeters, specifically designed by NAGRA (Swiss national technical competence centre in the field of deep geological disposal of radioactive waste) for validation of the BS activation predictions. Assessment of the 'representativity' of the selected experimental information for validation of the BS irradiation calculations was done on the basis of the ND-related Pearson correlation coefficients (in a similar manner as commonly used in criticality safety with ORNL SCALE/TSUNAMI software, see, e.g., discussions in $[4,8])$. The calculations were done using the ENDF/B-VII.1-based ACE files library.

\section{PSI METHODOLOGY FOR LWR DOSIMETRY AND ACTIVATION ASSESSMENTS}

A detailed description of the CASMO/SIMULATE/MCNP calculation methodology originally established at PSI for LWR dosimetry applications can be found in [9]. The methodology is based on utilization of the dedicated 'SOURCE4MC' tool which allows translation of Studsvik's codes CASMO/SIMULATE calculation results on the power and nuclide concentrations' spatial distributions into MCNP (SDEF card) pin-wise neutron source specifications [10]. Recently, the CASMO/SIMULATE/MCNP system has been linked at PSI with the FISPACT-II inventory code with the help of another PSI tool 'COMPLINK' [11]. COMPLINK was designed for the representation of material data in MCNP (SERPENT) inputs. Thus, it allows automatized splitting of MCNP model cells on a user-defined 3-D mesh in the selected regions of interest, which facilitates coupling with 0-D FISPACT-II set of models for isotopic inventory calculations. The entire calculation scheme referred to as SOURCE4MC-X allows neutron fluxes normalization based on the actual operating history and their consequent transfer from the MCNP calculations to FISPACT-II for activation and $d p a$ (displacement per atom) evaluations. The overall calculation route is demonstrated in Fig. 1. Finally, the ORNL code ADVANTG is applied for automatic generation of weight windows for efficient variance reduction for the MCNP deep penetration calculations $[2,5,6]$, when the flux attenuation from the reactor core to the concrete BS can exceed ten orders of magnitude.

\footnotetext{
${ }^{1}$ see https://menp.lanl.gov for details on the MCNP® software trademark.
} 


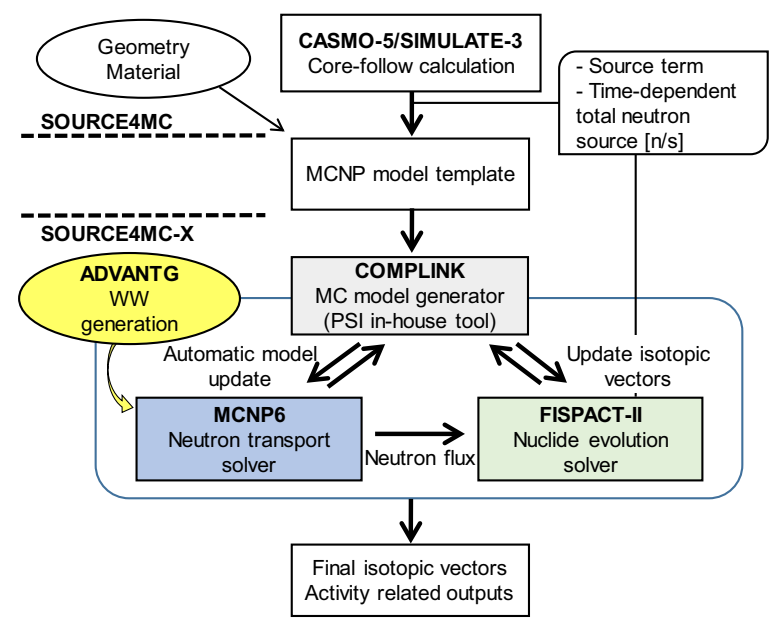

Figure 1. PSI methodology for LWR structures irradiation \& activation evaluations.

\section{NUCLEAR DATA STOCHASTIC SAMPLING WITH THE PSI TOOL NUSS}

The NUSS tool was developed to allow random sampling of the ND in ACE formatted files (read the reference ACE file and modify its data), based on uncertainties (covariances) information provided in ENDF-type libraries. At present, the ND can be sampled assuming the multivariate normal distributions, as soon as no solid grounds to utilize other types of probability density functions could be identified so far. For that, ENDF/B files MF-31, $-32,-33,-35$ need at first to be processed with NJOY to generate group-wise covariance matrices, which then can be used in the random sampling procedure applied to the continuous-energy ND [7]. NUSS also takes into account correlations between different reaction channels or isotopes, when such information is available. Fig. 2 shows an illustration of the NUSS approach. Each individual block of the total correlation matrix $(\mathrm{CM})$ on the left side of Fig. 2 consists of a $\mathrm{G}^{*} \mathrm{G}$ CM for individual reaction channel, where $G$ is the number of energy bins. In the present study, 187 energy bins structure was applied, consistently with the studies reported in [8]. The right side of Fig. 2 illustrates a point-wise cross-section and its group-wise uncertainty. One can notice that NUSS represents a nonintrusive approach for Monte Carlo codes (MCNP, SERPENT, RMC, etc.), which allows consistent comparison to other stochastic or deterministic methods, as was demonstrated previously [12]. By present time, NUSS was tested primary in combination with MCNP and SERPENT codes and applied for both criticality safety [4] and dosimetry applications $[8,13]$.
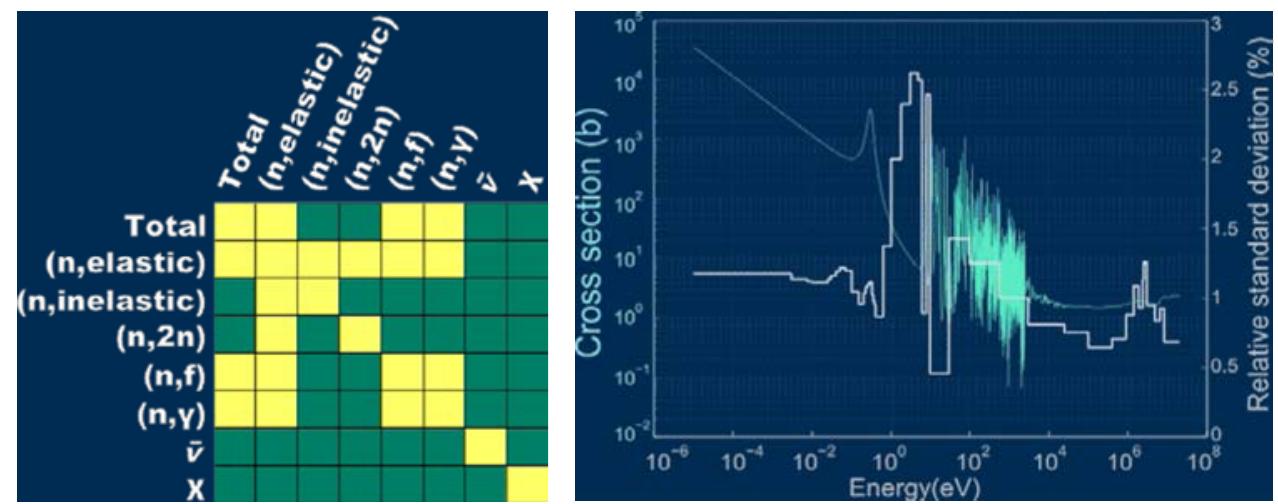

Figure 2. An illustration on the NUSS ND sampling approach (left - the total CM; right - a cross-section and its group-wise standard deviation). 


\section{THE SWISS PWR APPLICATION CASE}

The present study was done in application to the Swiss 'Pre-convoy' 3-loops PWR at the Goesgen NPP (Kernkraft Gösgen, KKG). The KKG MCNP model was originally developed at PSI for the Reactor Pressure Vessel (RPV) FNF analysis with the primary focus on the belt-line RPV region $[9,14]$ and efficient FNF predictions [15] and later extended on the ex-core neutron detector responses analyses [16].

The present model covers the concrete BS parts, keeping however the axial elevation limited by the core height and nearby 'axial reflectors'. The MCNP model is illustrated on Fig. 3, where the correspondence with the full-scale RPV scheme is also provided for clarity. It shall be noticed that for detailed materials activation studies one needs to use a full-scale 3D model, as is the case in the dedicated simulations of such type, performed at NAGRA [1,2]. However, for the sake of the given scoping study, it is considered adequate to use the present 'truncated' MCNP model. The MCNP model was complemented with cyclespecific coolant density specifications as well as with the 3D pin-wise neutron source specifications generated on the basis of the CASMO/SIMULATE core-follow calculations [9,10]. The model was validated using the experimental data available for the given PWR, as described in the next section.

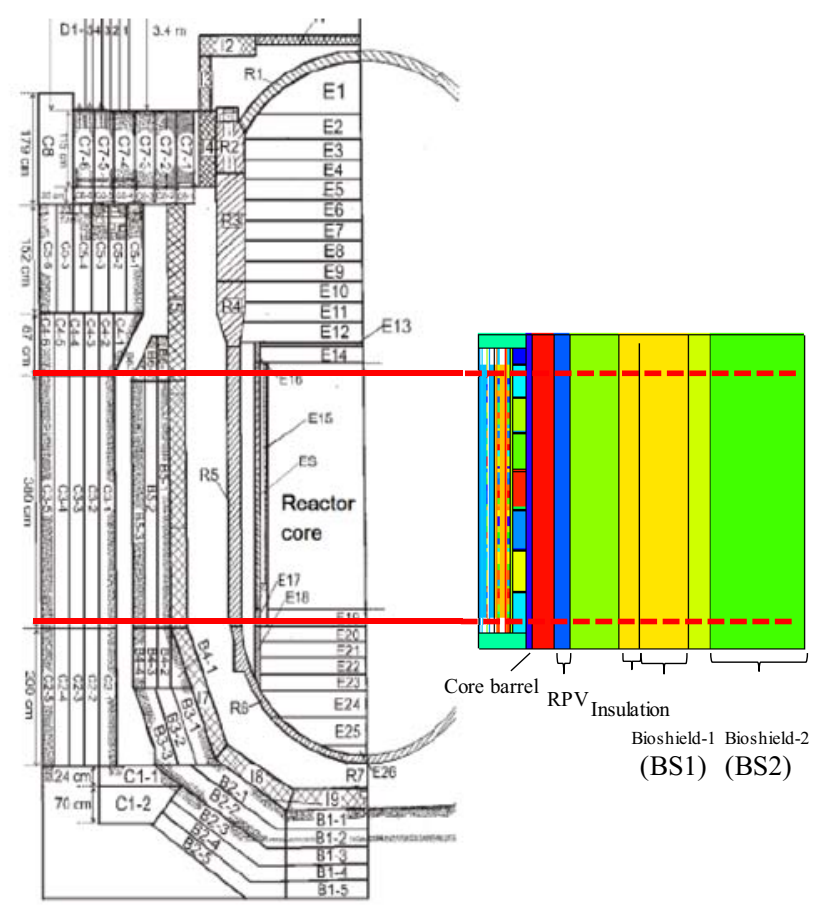

Figure 3. An illustration on the axial cross-section of the employed MCNP PWR model.

\section{DATA AVAILABLE FOR VALIDATION}

\subsection{Ex-core Dosimetry Data from KKG PWR}

The reference experimental information included the so called 'scraping test' (ST) data, which was obtained from activity measurements for the RPV steel scraping samples extracted after 11 and 27 operation cycles $[9,14]$ and also from activity measurements for the 'gradient probes' (GP) samples, extracted after irradiation during the first 27 operation cycles of the given PWR [14]. Locations of the considered samples within the used MCNP model are illustrated on Fig. 4. Detailed descriptions of the realized validation studies and the experimental data can be found in $[5,9,14]$. The dosimetry reactions used for experimental analysis were ${ }^{54} \mathrm{Fe}(\mathrm{n}, \mathrm{p}){ }^{54} \mathrm{Mn}$ and ${ }^{93} \mathrm{Nb}\left(\mathrm{n}, \mathrm{n}^{\prime}\right)^{93 \mathrm{~m}} \mathrm{Nb}$. 


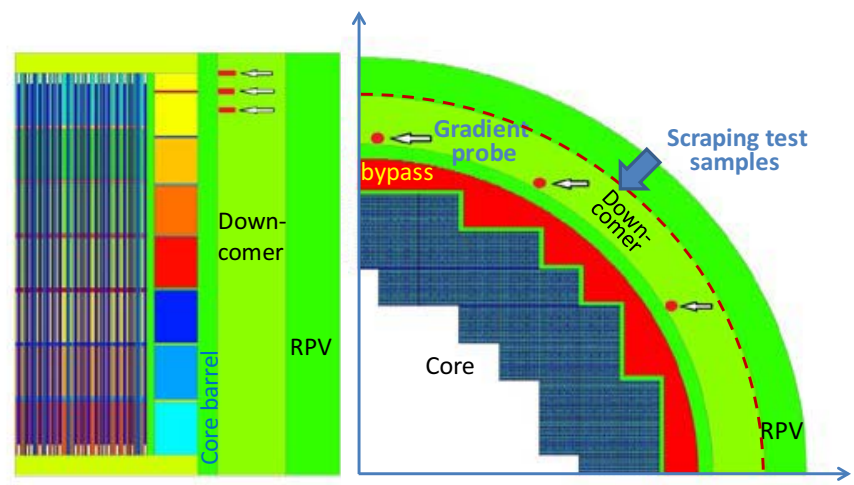

Figure 4. An illustration on the experimental data available for the FNF predictions validation.

\subsection{NAGRA's Dosimetry Data for Validation of the BS Activation Simulations}

As concerns the data appropriate for validation of the ex-RPV calculations, NAGRA has realized in collaboration with the KKG NPP a dedicated experimental program [1]. The measurements were designed for validation of the activation calculations for the ex-vessel reactor materials, which are necessary for optimization of the future dismantling and decommissioning processes [2]. Namely, three types of dosimeters sensitive to different neutron energy spectrum parts had been used in the irradiation campaigns: ${ }^{109} \mathrm{Ag}(\mathrm{n}, \gamma){ }^{110 \mathrm{~m}} \mathrm{Ag},{ }^{59} \mathrm{Co}(\mathrm{n}, \gamma){ }^{60} \mathrm{Co}$ and ${ }^{58} \mathrm{Ni}(\mathrm{n}, \mathrm{p}){ }^{58} \mathrm{Co}$. The cross-sections of these dosimetry reactions are illustrated on Fig. 6, together with the cross-sections outlined in Section 5.1. The figure was produced with the OECD/NEA JANIS software (https://www.oecd-nea.org/janis/). Obviously, the ${ }^{58} \mathrm{Ni}(\mathrm{n}, \mathrm{p})$ reaction is also appropriate for FNF validation studies, while the other two are more relevant for validation of the activation calculations primary induced by thermal neutrons.

It shall be acknowledged that even though NAGRA's experimental campaign was specifically focused on validation of the neutron transport and activation calculations at the concrete BSs, there were some technical limitations affecting the experimental setup and consequent measurements. At first, the dosimeters irradiation could only be done at a few (ten) locations (see Fig. 4. in [1]), all of them being above the core axial elevation, i.e. no experimental data could be obtained for validation of the concrete irradiation at the axial level of the maximum neutron flux amplitude. At second, the dosimeters were irradiated within only one reactor cycle and thus strictly speaking the measured data cannot be fully representative for a longer-time reactor operating history (note that the half-life of ${ }^{58} \mathrm{Co}$ is only 70.8 days, meaning that the ${ }^{58} \mathrm{Ni}$ detector is not well suitable for irradiation times above one reactor cycle). Thus, a pertinent question at the given circumstances is whether the experimental data employed at PSI for the RPV FNF validation could also be of relevance for validation of FNF at the concrete BSs.
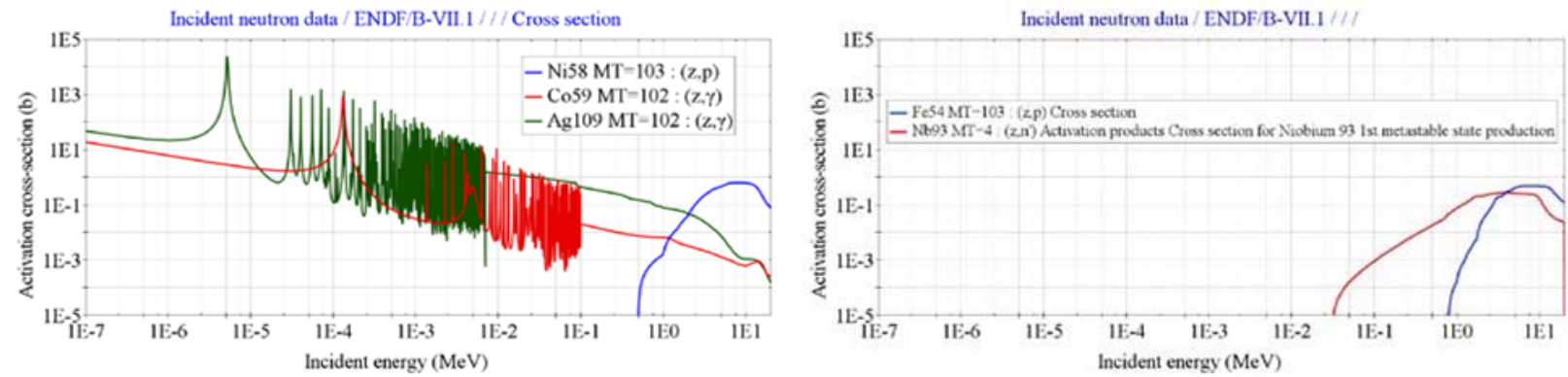

Figure 6. Dosimeters employed in Ref [1] for validation of the PWR BS activation simulations (left) and in Ref $[9,14]$ for validation of the PWR RPV FNF simulations (right). 


\section{RESULTS}

The calculations of the described MCNP models were realized with 250 random sets of the ACEformatted files for the relevant isotopes of the fuel, coolant and structural materials ${ }^{2}$, generated with the help of NUSS. The perturbed reactions included $(n, n),\left(n, n^{\prime}\right),(n, 2 n)$ and $(n, \gamma)$ cross-sections for nonactinides and in addition $(n, f)$, prompt neutron multiplicity $\overline{v_{p}}$ and prompt fission neutron spectra $\chi_{p}$ for actinides. ENDF/B-VII.1 library was used as the source of the underlying ND, including the related covariance matrices. Typical computation time of a single MCNP run with $10^{8}$ neutron histories using 20 cores of a high-performance PSI Linux cluster was $\sim 1$ hour (wall clock). The calculation results were processed to evaluate the ND-related uncertainties (see Table I) and the Pearson correlation coefficients, together with associated 95\% confidence intervals (see Table II). The data processing was done with the help of the "PerformanceAnalytics" package for R software environment (see for details https://cran.rproject.org/). The neutron flux results " $\Phi$ " (up to $20 \mathrm{MeV}$ ) were split in three energy bins with the boundaries at 0.1 and $1.0 \mathrm{MeV}$. The labels BS1 and BS2 correspond to the two BS areas as illustrated on Fig. 3. The 95\% confidence intervals are given in Table II below the Pearson coefficients values.

Table I. ND-related calculation uncertainties and corresponding MCNP relative errors ( $\left.\sigma^{\mathrm{MC}}, \%\right)$.

\begin{tabular}{|c|c|c|c|c|c|c|c|c|}
\hline & \multicolumn{8}{|c|}{ Dosimeter type / Location } \\
\hline & \multicolumn{2}{|c|}{ GP/Barrel } & \multicolumn{2}{|c|}{ ST / RPV } & \multicolumn{2}{|c|}{ BS1 } & \multicolumn{2}{|c|}{ BS2 } \\
\hline & $\sigma^{\mathrm{ND}}$ & $\sigma^{\mathrm{MC}}$ & $\sigma^{\mathrm{ND}}$ & $\sigma^{\mathrm{MC}}$ & $\sigma^{\mathrm{ND}}$ & $\sigma^{\mathrm{MC}}$ & $\sigma^{\mathrm{ND}}$ & $\sigma^{\mathrm{MC}}$ \\
\hline $\mathrm{Fe}-54(\mathrm{n}, \mathrm{p})$ & 6.6 & 0.2 & 11.0 & 0.2 & & & & \\
\hline $\mathrm{Nb}-93\left(\mathrm{n}, \mathrm{n}^{\prime}\right)$ & 7.0 & 0.1 & 10.8 & 0.1 & & & & \\
\hline Ag-109(n, $\gamma)$ & & & & & 10.2 & 0.2 & 16.7 & 0.3 \\
\hline 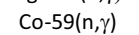 & & & & & 10.3 & 0.2 & 16.4 & 0.3 \\
\hline $\mathrm{Ni}-58(n, p)$ & & & & & 15.5 & 0.2 & 22.2 & 0.4 \\
\hline$\Phi \_\mathrm{E}<0.1 \mathrm{MeV}$ & 5.4 & 0.2 & 9.4 & 0.2 & 10.2 & 0.1 & 16.6 & 0.3 \\
\hline$\Phi \_E>0.1 \mathrm{MeV}$ & 6.0 & 0.1 & 9.9 & 0.1 & 15.3 & 0.2 & 19.0 & 0.3 \\
\hline $\bar{\Phi} \bar{\Phi}_{-} \mathrm{>}>1 \mathrm{MeV}$ & 7.4 & 0.1 & 11.5 & 0.1 & 10.4 & 0.1 & 16.9 & 0.3 \\
\hline dpa & 6.5 & 0.1 & 10.3 & 0.1 & 11.6 & 0.2 & 18.4 & 0.3 \\
\hline
\end{tabular}

Table II. ND-related Pearson correlation coefficients obtained for the considered models and parameters.

\begin{tabular}{|c|c|c|c|c|c|c|c|c|c|c|c|c|c|}
\hline \multirow{5}{*}{$\Phi \_B S 1 \_E<0.1 \mathrm{MeV}$} & \multicolumn{12}{|c|}{ Dosimeter type / Location } & \\
\hline & \multicolumn{2}{|c|}{ GP/Barrel } & \multicolumn{2}{|c|}{ ST/RPV } & \multicolumn{2}{|c|}{ RPV } & \multicolumn{3}{|c|}{ BS1 } & \multicolumn{3}{|c|}{ BS2 } & \\
\hline & Fe-54 & $\mathrm{Nb}-93$ & Fe-54 & $\mathrm{Nb}-93$ & FNF & $\mathrm{dpa}$ & $\mathrm{Ag}-109$ & Co-59 & $\mathrm{Ni}-58$ & $\mathrm{Ag}-109$ & Co-59 & $\mathrm{Ni}-58$ & \\
\hline & 0.91 & 0.87 & 0.91 & 0.96 & 0.97 & 0.98 & 1.00 & 1.00 & 0.76 & 0.87 & 0.88 & 0.65 & \\
\hline & {$[0.89,0.93]$} & {$[0.83,0.89]$} & {$[0.88,0.92]$} & ] $[0.95,0.97]$ & {$[0.96,0.97]$} & {$[0.98,0.99]$} & {$[1.00,1.00]$} & ] $[0.99,1.00]$ & {$[0.71,0.81]$} & {$[0.84,0.90][$} & {$[0.85,0.90]$} & {$[0.57,0.71]$} & \\
\hline \multirow[t]{2}{*}{$\Phi \_B S 2 \_E<0.1 \mathrm{MeV}$} & 0.73 & 0.65 & 0.88 & 0.86 & 0.83 & 0.87 & 0.87 & 0.86 & 0.90 & 1.00 & 1.00 & 0.85 & \\
\hline & {$[0.67,0.78]$} & {$[0.58,0.72]$} & {$[0.85,0.91]$} & ] $[0.83,0.89]$ & {$[0.79,0.86]$} & {$[0.84,0.90]$} & {$[0.84,0.90]$} & ] $[0.83,0.89]$ & {$[0.87,0.92]$} & {$[1.00,1.00][$} & {$[1.00,1.00]$} & ] $[0.81,0.88]$ & \\
\hline \multirow[t]{2}{*}{$\Phi \_B S 1 \_E>0.1 \mathrm{MeV}$} & 0.87 & 0.86 & 0.88 & 0.96 & 0.93 & 0.97 & 0.98 & 0.97 & 0.76 & 0.87 & 0.88 & 0.64 & \\
\hline & {$[0.84,0.90]$} & {$[0.82,0.89]$} & {$[0.85,0.90]$} & ] $[0.95,0.97]$ & {$[0.91,0.94]$} & {$[0.96,0.98]$} & {$[0.98,0.99]$} & ] $[0.96,0.98]$ & {$[0.70,0.80]$} & {$[0.84,0.90][$} & {$[0.84,0.90]$} & ] $[0.57,0.71]$ & 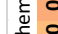 \\
\hline \multirow[t]{2}{*}{$\Phi \_B S 2 \_E>0.1 \mathrm{MeV}$} & 0.71 & 0.60 & 0.90 & 0.83 & 0.82 & 0.85 & 0.83 & 0.81 & 0.93 & 0.99 & 0.97 & 0.89 & \\
\hline & {$[0.65,0.77]$} & {$[0.52,0.67]$} & {$[0.87,0.92]$} & ] $[0.79,0.87]$ & {$[0.77,0.85]$} & {$[0.81,0.88]$} & {$[0.79,0.87]$} & {$[0.77,0.85]$} & {$[0.91,0.94]$} & {$[0.98,0.99][$} & {$[0.96,0.98]$} & {$[0.86,0.91]$} & \\
\hline \multirow[t]{2}{*}{ Ф_BS1_E>1MeV } & 0.70 & 0.77 & 0.76 & 0.90 & 0.69 & 0.86 & 0.82 & 0.81 & 0.75 & 0.82 & 0.82 & 0.64 & \\
\hline & {$[0.63,0.76]$} & {$[0.74,0.83]$} & {$[0.71,0.81]$} & {$[0.88,0.92]$} & {$[0.62,0.75]$} & {$[0.82,0.89]$} & {$[0.77,0.86]$} & {$[0.76,0.85]$} & {$[0.69,0.80]$} & {$[0.77,0.85][$} & {$[0.78,0.86]$} & {$[0.56,0.71]$} & \\
\hline \multirow[t]{2}{*}{$\Phi \_B S 2 \_E>1 \mathrm{MeV}$} & 0.69 & 0.56 & 0.90 & 0.81 & 0.78 & 0.82 & 0.79 & 0.77 & 0.95 & 0.97 & 0.94 & 0.94 & \\
\hline & {$[0.62,0.75]$} & {$[0.47,0.64]$} & {$[0.88,0.92]$} & {$[0.76,0.85]$} & {$[0.73,0.83]$} & {$[0.78,0.86]$} & {$[0.74,0.83]$} & {$[0.72,0.82]$} & {$[0.94,0.96]$} & {$[0.96,0.97][$} & {$[0.93,0.95]$} & ] $[0.93,0.96]$ & \\
\hline \multirow[t]{2}{*}{ dpa_BS1 } & 0.84 & 0.86 & 0.86 & 0.97 & 0.88 & 0.96 & 0.96 & 0.95 & 0.77 & 0.87 & 0.88 & 0.65 & \\
\hline & {$[0.80,0.87]$} & {$[0.83,0.89]$} & {$[0.83,0.89]$} & ] $[0.96,0.98]$ & {$[0.85,0.90]$} & {$[0.95,0.97]$} & {$[0.95,0.97]$} & {$[0.94,0.96]$} & {$[0.71,0.81]$} & {$[0.85,0.90][$} & {$[0.84,0.90]$} & ] $[0.58,0.72]$ & \\
\hline \multirow[t]{2}{*}{ dpa_BS2 } & 0.70 & 0.58 & 0.90 & 0.82 & 0.80 & 0.84 & 0.81 & 0.79 & 0.95 & 0.98 & 0.96 & 0.93 & \\
\hline & {$[0.64,0.76]$} & {$[0.50,0.66]$} & {$[0.88,0.92]$} & {$[0.78,0.86]$} & {$[0.75,0.84]$} & {$[0.80,0.87]$} & {$[0.77,0.85]$} & {$[0.74,0.84]$} & {$[0.93,0.96]$} & {$[0.97,0.98]$} & {$[0.95,0.97]$} & {$[0.91,0.94]$} & \\
\hline
\end{tabular}

The obtained results at first demonstrate a significant impact of the ND uncertainties on the dosimetry calculations, especially at the distant BS locations (note that the present ND uncertainty estimations can be significantly underestimated [17]). Secondly, they show that the reaction rates of NAGRA's dosimeters can be highly correlated with the BSs' FNF and $d p a$ calculation predictions, meaning that

\footnotetext{
2 The list of the most relevant isotopes treated by NUSS, in the MCNP ZAID format, included: 1001, 5011, 8016, 12024, 12025, 12026, 13027, 14028, 14029, 14030, 19041, 22046, 22047, 22048, 22049, 22050, 24050, 24052, 26054, 26056, 26057, 27059, 28058, 28060, 40090, 40091, 40092, 40094, 40096, 42092, 42094, 42095, 42096, 42097, 42098, 47109, 92235, 92238, 94239.
} 
NAGRA's data is relevant for validation of not only the activation calculations, but also the FNF/dpa ones. At the same time, some combinations of the results on $\Phi \_B S 1 \_E>1 \mathrm{MeV}$, $\Phi$ BS2_E $>1 \mathrm{MeV}$, dpa_BS1, dpa_BS2 and the GP, ST dosimeters' reaction rates also have relatively high correlations, which makes the GP and ST experimental data applicable for validation of the BS FNF and dpa calculations. One can also note that the GP and ST data is relatively highly correlated with the BS fluxes below $0.1 \mathrm{MeV}$. Thus, the GP and ST experimental data potentially can also be appropriate for validation of the thermal neutron flux predictions in the BS concrete. To justify this proposition, a more detailed analysis with refined energy bins' structure should be done. Note that the GP and ST locations correspond to the axial elevation of highest radiation doses, which are of major relevance for the BS activation studies.

\section{CONCLUSIONS}

Apart from the problem of the RPV irradiation induced embrittlement, one of the concerns related to the extended reactor operation times is the behaviour of the (reinforced) concrete reactor structures, including those supporting the RPV, under high FNF (specifically -under low flux/long irradiation time) conditions [3]. Not only the lack of experimental data for the materials behaviour under extended radiation exposures complicates the situation, but also the accuracy of the calculation simulations needs revision since for the more distant than RPV ex-core locations, the calculation biases can be significantly amplified due to the complex radiation streaming paths and increased importance of the neutron scattering processes [17]. Therefore, appropriate validation studies for the ex-vessel neutron transport simulations become an important task, in addition to the traditional RPV FNF assessments. The amount of high quality experimental data available for validation of the calculation methodologies and associated ND libraries is generally limited for majority of practical applications. This is also the case for such tasks as the reactor BS FNF, $d p a$ and/or activation predictions. Therefore, different kinds of available measurements, even if originally designed for other purposes, shall be re-assessed from the viewpoint of their applicability for validation of, e.g., the above listed types of simulations.

In the present work, the representativity of the available measured data for validation of the calculation parameters of interest was realised on the basis of the ND-related Pearson correlation coefficients assessment. The correlations between the calculation parameters (e.g., FNF) and measured data (specific activities) were analysed by applying the NUSS tool to randomly sample the ND used for the neutron transport simulations. Since the ND is one of the most important sources of uncertainties in the reactor dosimetry calculations, the obtained Pearson correlation coefficients can be considered as quantitative measures of similarity between the application type calculations and the validation studies, i.e. as a measure of representativity of the experimental data for the given application case.

The obtained results indicate that for the validation of the PWR BS FNF or $d p a$ assessments, both the experimental data obtained at NAGRA for the activation-oriented dosimeters, as well as the data originally obtained for the RPV FNF assessments and used for validation studies at PSI $[9,14]$ are valid. Noting the limited amount of the dosimeters and the FNF monitors irradiated at different locations, one can actually conclude that the available sets of the experimental data should be complementing each other. For future works, it is proposed to extend the study by inclusion into analysis of publicly open OECD/NEA Sinbad benchmarks and to verify their applicability for validation of simulations of LWR concrete BS irradiation. As well, extension of the random calculations' sample size and testing other ND libraries (ENDF/B-VIII.0 and JEFF-3.3) should be considered.

\section{ACKNOWLEDGMENTS}

This work was partly supported by swissnuclear, the association of the Swiss nuclear power station operators, through the PSI/SN R\&D project LRT-01 (2018-2019). The authors are grateful to the anonymous reviewers for their valuable suggestions. 


\section{REFERENCES}

1. B. Volmert, M. Pantelias, R.K. Mutnuru, E. Neukaeter, and B. Bitterli, "Validation of MCNP NPP Activation Simulations for Decommissioning Studies by Analysis of NPP Neutron Activation Foil Measurement Campaigns," EPJ Web of Conferences 106, 05010 (2016).

2. V. Bykov, S. Mosher, B. Volmert, A. Scolaro, M. Pantelias, A. Pautz, "NAGRA Activation Analysis for the Optimization of NPP Decommissioning and Component Segmentation Strategy," Proceedings of Int. Conf. PHYSOR 2018, Cancun, Mexico, April 22-26 (2018).

3. I. Remec, T. M. Rosseel, K.G. Field \& Y. Le Pape, "Radiation-Induced Degradation of Concrete in NPPs," ASTM 16th International Symposium on Reactor Dosimetry (ISRD-16), Sante Fe, New Mexico, USA, 05.07.2017- 05.12.2017. doi:10.1520/STP160820170059 (2018).

4. A. Vasiliev, D. Rochman, M. Pecchia, H. Ferroukhi, "On the options for incorporating nuclear data uncertainties in criticality safety assessments for LWR fuel," Ann. Nucl. Energy 116, pp. 57-68 (2018).

5. B. Jung, "PWR structures activation forecasts using Monte Carlo neutron transport simulations," Master Thesis, ETHZ/EPFL/PSI Master of Nuclear Engineering (2018).

6. B. Jung, A. Vasiliev, M. Pecchia, H. Ferroukhi, "Optimization and sensitivity studies towards PWR structures activation characterization using the MCNP code," ANS Students Conference 2018, University of Florida, Gainesville FL, USA, April 5-7, 2018 (2018).

7. T. Zhu, A. Vasiliev, H. Ferroukhi, A. Pautz, "NUSS: A tool for propagating multigroup nuclear data covariances in pointwise ACE-formatted nuclear data using stochastic sampling method," Ann. Nucl. Energy, 75, pp. 713-722 (2015).

8. A. Vasiliev, D. Rochman, M. Pecchia, H. Ferroukhi, "Exploring Stochastic Sampling in Nuclear Data Uncertainties Assessment for Reactor Physics Applications and Validation Studies," Energies 2016 (9, 1039), pp. 1-18 (2016).

9. A. Vasiliev, H. Ferroukhi, M.A. Zimmermann, R. Chawla, "Development of a CASMO4/SIMULATE-3/MCNPX Calculation Scheme for PWR Fast Neutron Fluence Analysis and Validation against RPV Scraping Test Data,” Ann. Nucl. Energy 34(8), pp. 615-627 (2007).

10. H. Ferroukhi, K. Hofer, J-M. Hollard, A. Vasiliev, M.A. Zimmermann, "Core Modelling and Analysis of the Swiss Nuclear Power Plants for Qualified R\&D Applications," Proceedings of Int. Conf. PHYSOR'08, Interlaken, Switzerland, September 14-19 (2008).

11. M. Pecchia, J. J. Herrero, H. Ferroukhi, A. Vasiliev, S. Canepa, A. Pautz, "COMPLINK: A Versatile Tool for Automatizing the Representation of Material Data in MCNP Models," Proceedings of Int. Conf. Nuclear Criticality Safety, ICNC 2015, Charlotte, NC, USA, September 13-17 (2015).

12. D. Rochman, A. Vasiliev, H. Ferroukhi, T. Zhu, S.C. van der Marck, A.J. Koning, "Nuclear data uncertainty for criticality-safety: Monte Carlo vs. linear perturbation," Ann. Nucl. Energy, 92, pp. 150-160 (2016).

13. A. Vasiliev, H. Ferroukhi, T. Zhu, A. Pautz, "Nuclear Data Library Effects on Fast to Thermal Flux Shapes around PWR Control Rod Tips," Nuclear Data Sheets 118, pp. 575-578 (2014).

14. A. Dupré, A. Vasiliev, H. Ferroukhi, A. Pautz, "Towards modeling and validation enhancements of the PSI MCNPX fast neutron fluence computational scheme based on recent PWR experimental data," Ann. Nucl. Energy 85, pp. 820-829 (2015).

15. A. Vasiliev, H. Ferroukhi, E. Kolbe, M.A. Zimmermann, "On the use of importance factors from Monte Carlo calculations for efficient fast neutron fluence prediction," Proceedings of Int. Conf. PHYSOR'08, Interlaken, Switzerland, September 14-19 (2008).

16. M. Pecchia, A. Vasiliev, H. Ferroukhi, A. Pautz, "A methodology for evaluating weighting functions using MCNP and its application to PWR ex-core analyses," Ann. Nucl. Energy 105 pp. 121-132 (2017).

17. A. Vasiliev, D. Rochman, M. Pecchia, H. Ferroukhi, "On the Importance of the Neutron Scattering Angular Distributions for the LWR Fast Neutron Dosimetry," Proceedings of Int. Conf. PHYSOR 2018, Cancun, Mexico, April 22-26 (2018). 